\title{
Art of Reading an Article in the Journal
}

\author{
Geetha Balsarkar ${ }^{1}$
}

Received: 21 December 2021 / Accepted: 21 December 2021 / Published online: 21 January 2022

(c) Federation of Obstetric \& Gynecological Societies of India 2022

"One swallow doesn't make a summer" This is a famous quote by Aristotle.

Reading scientific literature and making sense of the journal articles is important for researchers and clinicians. With an overflow of medical journals available, and similar articles published in various journals it is essential to develop a method to choose and read and interpret the right articles. The results and conclusion can then be translated in clinical practice. At the beginning, the journal has to be chosen and the concerned article selected. For a logical and step by step approach to reading a scientific manuscript, there are ten steps. With these steps, one should be able to read a scientific article with ease. The reader should begin by reading the title, abstract and conclusions first. If it appeals to the scientific mind, a decision is made to read the entire article. One can read and appreciate a scientific manuscript if a systematic approach is followed in a simple and logical manner.

It has become important for the clinicians and students to read articles from scientific journals. Some of the journals are in the curriculum of postgraduate students. Scientific reading helps us to keep abreast of what progress is happening in the speciality we are trained in and also makes us aware of current trends in providing optimum health care to our patients. Reading scientific literature is a compulsory for students interested in research, for choosing their topics of research and carrying out their experiments.

"There is no problem that a library card can't solve" according to author Eleanor Brown.

Sackett [1] and Durbin [2] suggested common reasons why most of us read journal articles and these are listed in Table 1.

The scientific literature today is growing at an exponential rate, especially in the field of Obstetrics and gynaecology,

\section{Geetha Balsarkar: Editor in Chief.}

\section{Dr. Geetha Balsarkar}

gdbalsarkar@yahoo.com

1 Seth G.S. Medical College and Nowrosjee Wadia Maternity Hospital, Mumbai, India during Covid times and post Covid era. What was true last year is not true for Covid management anymore. The strains have evolved, the drugs used are different and the presentation is different and management too. Many similar clinical situations have evolved over the years like small pox, tetanus, prematurity, etc.

Between the years 1978 and 1985, approximately 272,344 articles were published annually as listed in Medline. Between 1986 and 1993, this number however reached 344,303 articles per year, and between 1994 and 2001, the figure has grown to 398,778 articles per year [3]. To be updated with complete current knowledge and recent data, a physician practicing general medicine has to read 17 articles a day, 365 days a year [4]. Obstetrics and gynaecology being a very progressive branch with various advances in endoscopy, vaginal surgery, assisted reproductive techniques and more, this number will be even more.

During the prolonged lockdown of Covid-19, the number of articles being submitted to journals has almost doubled. Reading of scientific literature has also increased exponentially during lockdown. The rapidly changing scenario of Covid has also necessitated increased reading of scientific literature. The internet has rapidly become a quick and accessible source of obtaining information in whatever subject is required, more so due to lockdown. However reading journal articles from reputed journals, whether from print or online still remains the most common way of acquiring correct and quick information for clinicians.

Not all research articles published whether online or print are clinically applicable and relevant, and it is important to decide whether the quality of the study is good to warrant reading of the article in depth.

The first step for a reader is to choose a right article for reading, depending on one's individual requirement. The next step is to read the selected article methodically and efficiently [5]. A simple decision-making flow chart is depicted in Fig. 1, which helps one to decide the type of article to select.

On opening the journal, different people have different perception. For some an article might appear very difficult, 
Table 1 Common reasons for reading Journal articles
To update oneself with progress in a particular speciality/field of study

To find out a solution for a specific problem-could be diagnostic (texts/methods) or therapeutic (medical/surgical)

To know about causation, clinical features, and course of a disorder/disease

To understand certain fundamental aspects like pathophysiology

To get an idea for carrying out a research work

The article has been assigned to be read (for e.g., by an instructor to a postgraduate student) To find support for one's views

To impress others

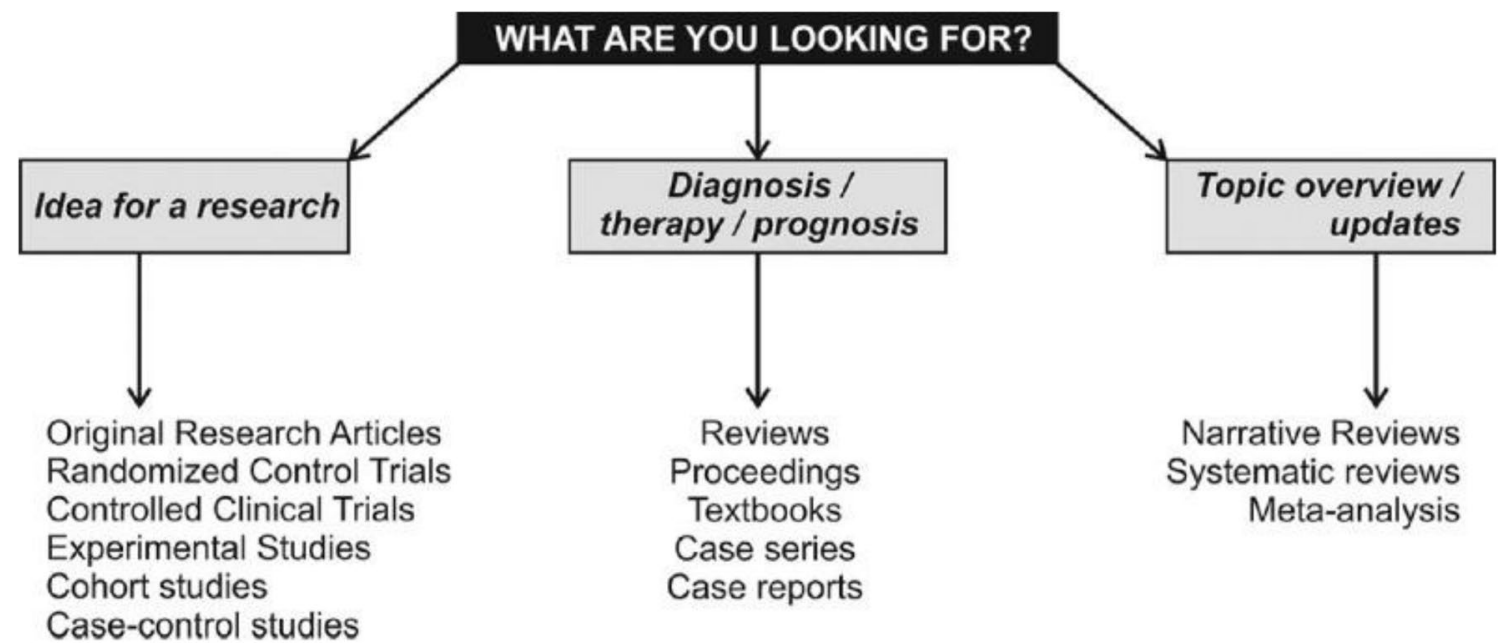

Fig. 1 Which article is to be read from the journal? [5]

boring or meaningless or confusing for others with its methodology, tables and graphs. Assimilating and understanding a research article in a short span of time can be a frustrating experience, especially for the one who does not know the art of reading scientific literature. This article is to make reading a pleasant experience for the readers.

Most scientific articles are organized as follows: $[2,5,6]$.

Title: Topic and information about the authors along with their title and organisation of research.

\section{Abstract}

Brief overview of the article which may impress the reader to read the article.

Introduction: Background information as to why this particular topic was chosen and statement of the research hypothesis.

Methods: Details of how the study was conducted, procedures followed, instruments used and variables measured.

Results: All the data of the study along with figures, tables and/or graphs.
Discussion: The interpretation of the results and implications of the study in clinical practice.

References/Bibliography: Citations of sources from where the information was obtained.

Review articles are by experts in the field and may or may not usually follow the above pattern, as they might include several studies unless they are systematic reviews or metaanalysis [7-9].

The cardinal rule is: Never start reading an article from the beginning to the end.

For a logical and step by step approach to reading a scientific manuscript, there are ten steps. With these steps, one should be able to read a scientific article with ease. The reader should begin by reading the title, abstract and conclusions first. If it appeals to the scientific mind, a decision is made to read the entire article. One can read and appreciate a scientific manuscript only if a systematic approach is followed in a simple and logical manner. Sometimes it is better to begin by identifying the conclusions of the study and then proceed by reading the title and the abstract. If the article does not have an abstract, read the conclusions or the summary at the end of the article first. After reading the abstract 


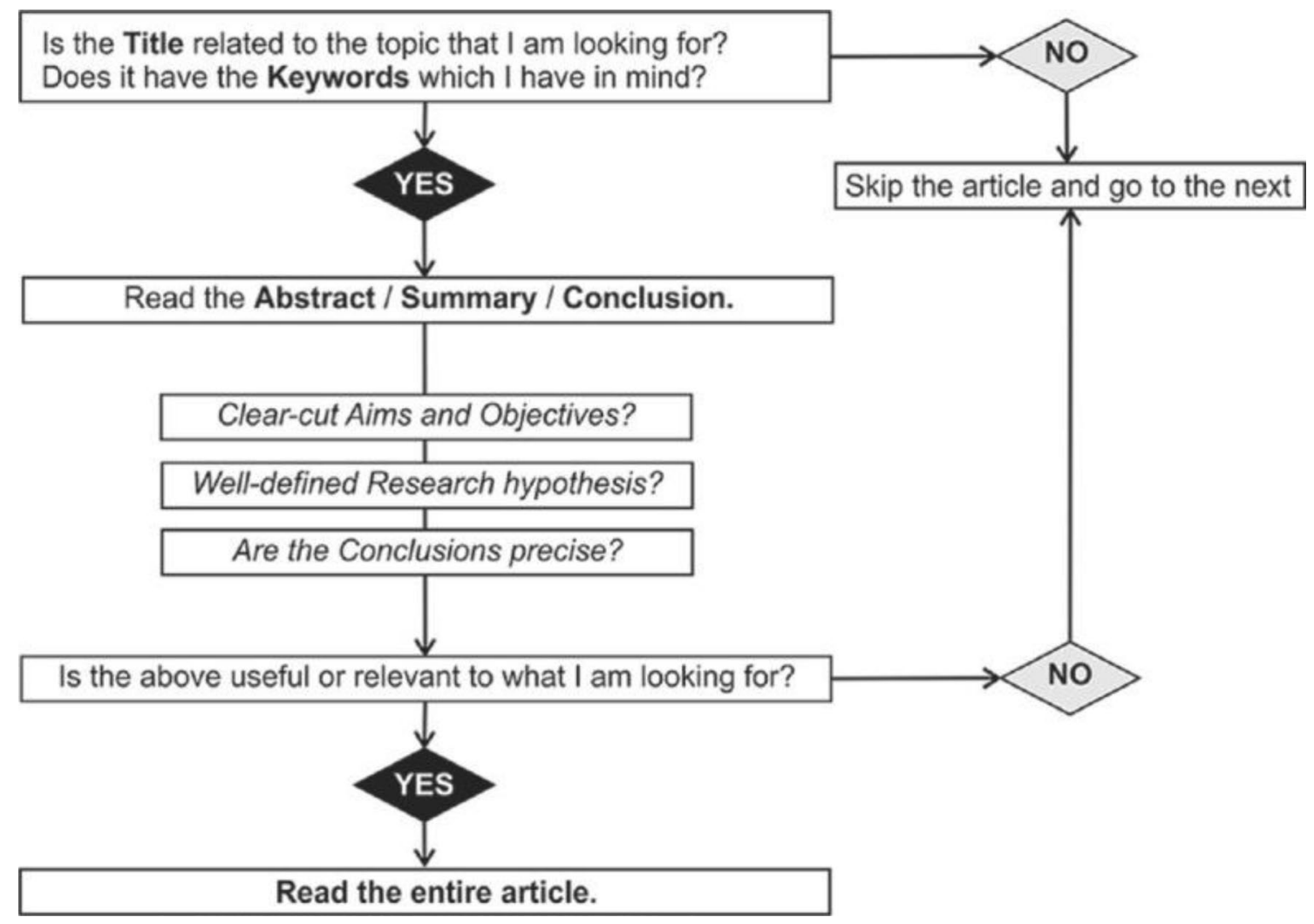

Fig. 2 Decision-making flow chart to decide whether to read the chosen article or not $[7,8]$

or conclusions, if the reader thinks it is interesting or useful, then the entire article can be read (Fig. 2).

\section{The Title}

The title of the article is the face of the article and it attracts a reader in the first place. A good title will inform the potential reader a great deal about the value of the study to decide whether to go ahead with the paper or dismiss it. Most readers prefer titles that are descriptive and self-explanatory without having to look at the entire article to know what it is all about [10]. For example, the paper entitled "Urine pregnancy test-A blessing for Obstetricians" gives an idea about the article in general to the reader. But there is no indication in the title whether it is a review article on Urine pregnancy test or an original research. If the title had been "Comparison of Urine pregnancy test and other modalities to diagnose pregnancy early and effectively", even a novice reader would have a better understanding of the content of the paper.

\section{Abstract}

Abstract helps us determine whether we should read the entire article or not. In fact, most journals provide abstract free of cost online allowing us to decide whether we need to purchase the entire article. Most scientific journals now have a structured abstract with separate subheadings like introduction (background or hypothesis), methods, results and conclusions making it easy for a reader to identify important parts of the study at one glance [11]. Moreover, there is usually a restriction about the number of words that can be included in an abstract. This makes the abstract concise enough for one to read rapidly.

The abstract can be read in a systematic way by answering certain fundamental questions like what was the study about, why and how was the study conducted, the results and their conclusions. The reader should make a note of any questions that were raised while reading the abstract and be sure that answers have been found after reading the entire article [12].

Once the reader has decided to read the entire article, one can begin with the introduction. 


\section{Introduction}

The purpose of the introduction is to provide the rationale for conducting the study. This section usually starts with existing knowledge and previous research of the topic under consideration. Typically, this section concludes with identification of gaps in the literature and how these gaps stimulated the researcher to design a new study $[5,12]$. A good introduction should provide proper background for the study. The aims and objectives of the study are usually mentioned at the end of the introduction. The reader should also determine whether a research hypothesis (study hypothesis) was mentioned and later check whether it was answered under the discussion.

\section{Materials and Methods}

This section gives the technical details of how the experiments were carried out. In most of the research articles, all details are rarely included but there should be enough information to understand how the study was carried out if some researcher decides to duplicate the study [12]. Information about the number of subjects included in the study and their categorisation, sampling methods, the inclusion criteria and exclusion criteria and the variables chosen can be derived by reading this section. The reader should get acquainted with the procedures and equipment used for data collection and find out whether they were appropriate.

\section{Results of the Study}

In this section, the researchers give details about the data collected, either in the form of figures, tables and/or graphs. Ideally, interpretation of data should not be reported in this section, though statistical analyses are also presented. The reader should meticulously go through this segment of the manuscript and find out whether the results were reliable (same results over time and can be duplicated) and valid (measure what it is supposed to measure). An important aspect is to check if all the subjects present in the beginning of the study were accounted for at the end of the study. If the answer is no, the reader should check whether any explanation for attrition was provided.

The results that were statistically significant and results that were not, must be identified. One should also observe whether a correct statistical test was employed for analysis and was the level of significance appropriate for the study. To appreciate the choice of a statistical test, one requires an understanding of the hypothesis being tested [10, 11]. Table 2 provides a list of commonly used statistical tests used in scientific publications.
Table 2 Statistical tests and their use in research

\begin{tabular}{ll}
\hline Test & How reported typically \\
$t$-test & $(t=2.79, p<.05)$ \\
& $\left(t_{.05,2,24}=2.79, p<.05\right)$ \\
& $p<.05$ \\
& $*$ Beside mean with notation on bottom \\
& of table \\
& As exact probability \\
& $F$-statistic \\
& $p$ value \\
ANOVA & Some kind of asterisk indication beside \\
-test & the means of $F$ with $p$ notation at bot- \\
& tom of table \\
& Might be stated in any of these ways \\
& within the text, or in a table of means \\
& Regression equation \\
Regression & Beta \\
& $R^{2}$ \\
Chi-squared test & Chi-squared statistic \\
& Degrees of freedom \\
& $p$ value \\
Wilcoxon signed-rank test & $Z$-statistic \\
Kruskal-Wallis & $p$ value \\
& H-statistic \\
& Degress of freedom \\
& $p$ value \\
\hline & \\
&
\end{tabular}

\section{Description and Interpretation}

Description and interpretation of these tests has to be in detail. It is not only important to know whether a difference or association is statistically significant but also appreciate whether it is large or substantial enough to be useful clinically [13]. In other words, what is statistically significant may not be clinically significant.

\section{Discussion}

This is the most important section of the article where the research questions are answered and the meaning of analysis and interpretation of the data are presented. Usually the study results are compared with other similar studies, explaining in what aspects they were different or similar. Ideally, no new data should be presented under discussion and no information from other sections should be repeated [14] In addition, this section also discusses the various strengths and limitations/ shortcomings of the study, providing suggestions about areas that need additional research.

The meaning of results and their analyses, new theories or hypotheses, limitations of the study, explanation of differences and similarities with other comparable studies, and suggestions for future research are offered in this section. One important point to remember is that the discussions are the 
authors' and co-authors' interpretations and opinions and not necessarily facts.

\section{Reading the Conclusion Again}

Though conclusion part had been read at the beginning to decide whether this paper has to be read, it is prudent to read it again in the end to confirm whether what we had inferred initially is correct. If the conclusion had not made sense earlier, it may make sense after having read through the entire article [15].

\section{Interpreting Conclusions}

Conclusions is what remains with the author long after the rest of the paper is forgotten. Conclusion also decides whether the statistical significance obtained is clinically significant or not. Typically, in conclusions we have to answer the five Cs [16].

Category: What type of paper is this? Is it an experimental study? A combined analysis of previous studies? An introduction of a new methodology?

Context: Which other literature papers is it related to? What were the theoretical bases that were used to analyse the problem?

Correctness: Are the assumptions valid?

Contributions: How does the paper contribute to the existing scientific understanding?

Clarity: Is the paper well-written and easy to understand?

\section{Critical Thinking and Stimulation for Further Reading}

Published papers are not truths etched in stone. Science is a never-ending work in progress, and it is essential that the reader pushes back against the author's interpretation to test the strength of their conclusions. Everyone has their own perspective and may interpret the same data in different ways. Mistakes are sometimes published, but more often these apparent errors are due to other factors such as limitations of a methodology and other limits to generalizability (selection bias, unaddressed or unappreciated confounders). When reading a paper, it is important to consider if these factors are pertinent.

Talking and discussing about an article that you have recently read in a journal club or more informal environment forces further active reading and participation. Teaching is one of the best ways to learn and that teachers learn the material even better as the teaching task becomes more complex anecdotally, such observations inspired the phrase "to teach is to learn twice."

Critical thinking is really a tough skill to learn but ultimately boils down to evaluating data while minimizing biases.

\section{References}

1. How to read clinical journals: I. Why to read them and how to start reading them critically. Can Med Assoc J. 1981;124:555-8.

2. Durbin CG. Jr How to read a scientific research paper. Respir Care. 2009;54:1366-71.

3. Druss BG, Marcus SC. Growth and decentralization of the medical literature: implications for evidence-based medicine. J Med Libr Assoc. 2005;93:499-501.

4. Davidoff F, Haynes B, Sackett D, Smith R. Evidence based medicine. BMJ. 1995;310:1085-6.

5. Subramanyam RV. Art of reading a journal article: methodically and effectively. J Oral Maxillofac Pathol. 2013;17(1):65-70. https://doi.org/10.4103/0973-029X.110733.

6. How to read a scientific paper. [Last accessed on 2011 Oct 9]. Available from: http://www.sciencebuddies.org/science-fair-proje cts/top_science-fair_how_to_read_a_scientific_paper.shtml.

7. Callcut RA, Branson RD. How to read a review paper. Respir Care. 2009;54:1379-85.

8. Greenhalgh T. Papers that summarise other papers (systematic reviews and meta-analyses). BMJ. 1997;315:672-5.

9. Akobeng AK. Understanding systematic reviews and meta-analysis. Arch Dis Child. 2005;90:845-8.

10. Peh WC, $\mathrm{Ng} \mathrm{KH}$. Basic structure and types of scientific papers. Singap Med J. 2008;49:522-5.

11. Hudson-Barr D. How to read a research article. J Spec Pediatr Nurs. 2004;9:70-2.

12. International Committee of Medical Journal Editors. Uniform requirements for manuscripts submitted to biomedical journals: writing and editing for biomedical publication. Available from: http://www.icmje.org/urm_main.html. Accessed 10 Oct 2011.

13. Das R, Das PN. Biomedical research methodology including biostatistical applications. New Delhi: Jaypee Brothers Medical Publications (P) Ltd; 2011. p. 123-45.

14. Hess DR. How to write an effective discussion. Respir Care. 2004;49:1238-44.

15. Riegelman RK. Studying a study and testing a test: how to read the medical evidence. 5th ed. Philadelphia: Lippincott Williams and Wilkins; 2005. p. 45.

16. Keshav S. How to read a paper. 2012. Retrieved from http://blizz ard.cs.uwaterloo.ca/keshav/home/Papers/data/07/paper-reading. pdf.

Publisher's Note Springer Nature remains neutral with regard to jurisdictional claims in published maps and institutional affiliations. 